\title{
RED DE ESTACIONES DE OBSERVACIÓN ATMOSFÉRICA EN LA ANTÁRTICA, UNA COLABORACIÓN INTERNACIONAL PARA LA INVESTIGACIÓN DEL CAMBIO CLIMÁTICO
}

\author{
STATIONS NETWORK FOR ATMOSPHERIC OBSERVATION IN ANTARCTICA, AN \\ INTERNATIONAL COLLABORATION FOR CLIMATE CHANGE RESEARCH
}

Jorge F. Carrasco ${ }^{1}$

\begin{abstract}
The Global Climate Observing System (GCOS) was established in 1992 by the World Meteorological Organization, the United Nation Environmental Program, the International Oceanography Commission and the International Council Scientific Union. This is as result of the Decision 11/CP.9 of the Fifth Conference of Parties of the Framework Convention for Climate Change of United Nation. GCOS is based on the compromise of the countries in maintaining the observing stations and providing the data. With this, GCOS accomplishes the goal of providing to the research community with a basic observing network for monitoring the climate system that will allow detecting the changes and responses of all components of the system. Antarctica is one of the regions for which GCOS has been essential for the observation and climate change research.
\end{abstract}

Key words: GCOS, climate observation, climate system, climate change.

\section{RESUMEN}

El Sistema Mundial de Observación del Clima fue establecido en 1992 por la Organización Meteorológica Mundial, el Programa Medioambiental de Naciones Unidas, la Comisión Oceanográfica Intergubernamental y el Concejo Internacional para la Ciencia. Esto como respuesta a la Decisión 11/ CP.9 de la Quinta Conferencia de las Partes de la Convención Marco sobre Cambio Climático de las Naciones Unidas. El SMOC se basa en el compromiso de los países en mantener en funcionamiento las estaciones de monitoreo y de proveer los datos observados. Con esto el SMOC cumple con el objetivo

1 Dirección Meteorológica de Chile, Av. Portales 3450, Estación Central, Santiago; Centro de Estudios Científicos, Av. Arturo Prat 540, Valdivia. jorcar59@gmail.com. 
de proveer a la comunidad científica con una red básica de observación y los datos necesarios para el monitoreo del sistema climático que permitan detectar los cambios y respuestas de todos los componentes del sistema. La Antártica es una de las regiones en la cual el SMOC ha sido esencial para la observación y la investigación del cambio climático.

Palabras clave: SMOC, observación del clima, sistema climático, cambio climático.

\section{INTRODUCCIÓN}

De acuerdo a los resultados de los modelos de simulación climática en las latitudes polares del hemisferio sur, en particular en las zonas costeras del continente antártico, muestran que un aumento en la temperatura del aire debería tener lugar para fines del siglo XXI (Meehl et al. 2007), induciendo a la vez a un aumento en la humedad, la nubosidad y la precipitación. Junto a estos cambios atmosféricos se esperan que ocurran cambios en los otros elementos del sistema climático como son el retroceso de glaciares, disminución de la formación y reducción de la extensión del hielo marino, desintegración de las plataformas de hielo permanente, así como también alteraciones en el comportamiento de la fauna y flora antártica. Una de las limitaciones de los estudios de cambio climático en las zonas polares del sur es la escasez de datos ambientales ya que la gran mayoría de las estaciones de observación comienzan a operar después de los años cincuenta y por otra parte, fueron en su mayoría instaladas en áreas costeras.
Prácticamente desde los inicios de la entrada en vigencia del Tratado Antártico, la Organización Meteorológica Mundial reglamentó la observación sistemática y las telecomunicaciones para la circulación y envío de las observaciones a los centros nacionales, regionales y mundiales de acopio de información meteorológica. Como antes, hoy en día la observación entra a los modelos numéricos de simulación para el pronóstico diario del tiempo, así como también, son la base para los estudios de variabilidad y cambio climático. La Red Básica de Estaciones de Superficie y la Red Básica de Altura (radiosondeos) en la Antártica es la que se muestra en la Figura 1. Estas estaciones cumplen con un programa de observaciones sinópticas de superficie de al menos 4 veces al día y la elaboración de un reporte climático mensual de las variables atmosféricas (CLIMAT). Las estaciones de la Red de Altura cumplen un programa de uno ó dos vuelos de radiosondeos diario y un resumen climático mensual (CLIMAT/TEMP). Basado en estas redes básicas y otras similares alrededor del planeta que incluyen a la vez observaciones terrestres y oceánicas, se a)

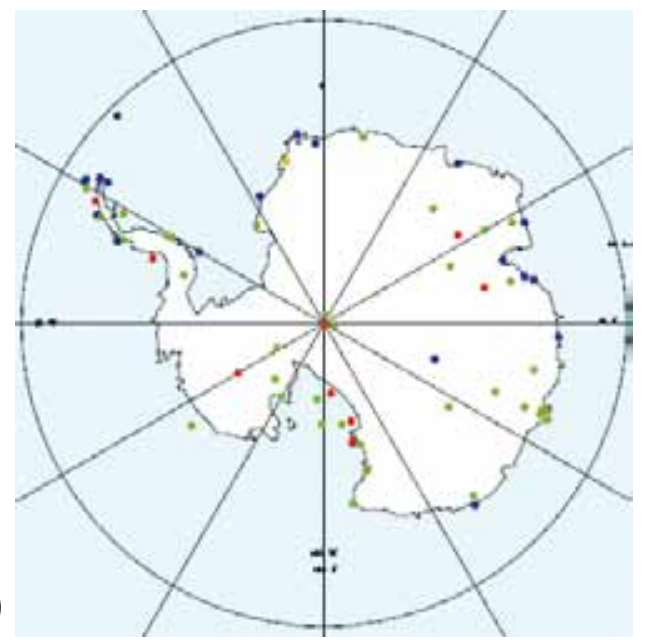

b)

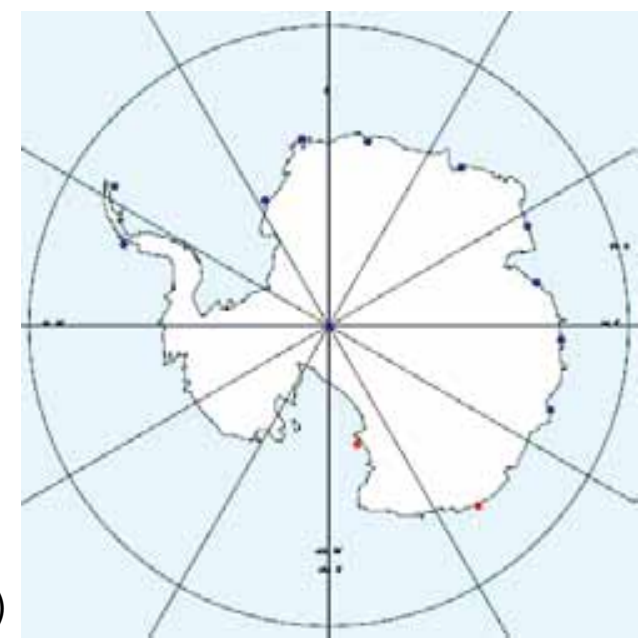

Fig. 1. Distribución de la Red Básica de Estaciones Meteorológicas de Superficie (a) y de la Red Básica de estaciones de Altura (b). 
implementa el Sistema Mundial de Observación del Clima (SMOC o en inglés GCOS: Global Climate Observing System). Brevemente se describe aquí lo que es el SMOC y se presentan dos ejemplos de cómo esta iniciativa, junto a otras, ayudan a la investigación del clima.

\section{SISTEMA MUNDIAL DE OBSERVACIÓN DEL CLIMA}

El SMOC fue establecido en 1992 por la Organización Meteorológica Mundial (OMM ó en inglés WMO: World Meteorological Organization), el Programa Medioambiental de Naciones Unidas (UNEP), la Comisión Oceanográfica Intergubernamental (IOC: International Oceanographic Commission) de UNESCO (United Nations Educational Scientific and Cultural Organization) y el Concejo Internacional para la Ciencia (ICSU: International Council for Science Union). Tiene por objetivo el proveer los datos necesarios para el monitoreo del sistema climático y detectar los cambios y respuestas de todos los componentes del sistema.

Por lo tanto, el SMOC incluye las propiedades y procesos físicos, químicos y biológicos tanto de la atmósfera, los océanos, de la criósfera, los hidrológicos y terrestres.

El SMOC incluye los diversos programas de observación que existen a nivel mundial, tales como, el Sistema de Observación Terrestre Mundial (GTOS: Global Terrestrial Observing System), el Sistema de observación de los Océanos (GOOS: Global Ocean Observing System) y de la Vigilancia del Tiempo Mundial (WWW: World Weather Watch) y los programas de vigilancia de la Atmósfera de la OMM.

En 1998 la tercera Conferencia de las Partes (COP) de la Convención Marco sobre Cambio Climático (FCCC: Framework Convention for Climate Change) de las Naciones Unidas (UNFCCC) reafirma la urgencia de tener una cobertura mundial de datos de variables atmosféricas, oceanográficas y terrestres; y la necesidad de revertir la continua degradación de la red existente. Más tarde, la Decisión 11/CP.9 de la COP5 invita a la secretaria del SMOC a realizar talleres regionales para analizar las capacidades existentes en los países, las necesidades de financiamiento y status de la red existente con el fin de revertir y superar las deficiencias y cobertura de las diversas redes que proveen dato sobre el monitoreo del sistema climático.

Los objetivos del SMOC son apoyar todos los componentes del Programa Mundial del Clima, la labor del Panel Intergubernamental de Cambio Climático (IPCC: Intergovernmental Panel on Climate Change) y las políticas internacionales de desarrollo que realiza la Convención Marco sobre Cambio Climático de las Naciones Unidas (UNFCCC); y en particular, proveer con observaciones del clima continuas y comprensivas con el fin de satisfacer necesidades para (GCOS 2007):

- Monitorear el sistema climático;

- Detección y atribución del cambio climático;

- Predicción climática operacional a escala estacional e interanual;

- Investigación para mejorar el entendimiento, la modelación y la predicción del sistema climático;

- Aplicaciones y servicios para el desarrollo económico sustentable;

- Estudios de impactos, vulnerabilidad y adaptación a la variabilidad natural del clima y al cambio climático de origen antropogénico; y

- Cumplir los requerimientos de los acuerdos de la UNFCCC y otras convenciones internacionales.

El Comité de Gestión del SMOC identificó una lista de variables esenciales para la observación del sistema climático y para el apoyo a la UNFCCC. Estas variables son técnica y económicamente viables y tienen un alto impacto con respecto a los requerimientos científicos para la observación sistemática. En la Tabla 1 se lista las distintas variables esenciales para la atmósfera, el océano y áreas terrestres (GCOS 2007).

Entre los principios establecidos para la formación de la Red SMOC están: la calidad y homogeneidad de los datos de las estaciones seleccionadas, que las estaciones cuenten con observaciones históricas ininterrumpidas y el mantenimiento operativo futuro de las mismas, que las consideraciones de las necesidades de estudios y productos del monitoreo climático medioambiental, como el informe del IPCC, deben ser prioridades integradas a la a nivel nacional, regional y global.

En consideración de los principios de monitoreo climático del SMOC, en la región antártica, un subconjunto de la Red Básica de Estaciones fueron seleccionadas para formar parte de la Secretaria 
Tabla 1. Variables esenciales del SMOC (GCOS 2007).

\begin{tabular}{|c|c|c|}
\hline DOMINIO & \multicolumn{2}{|c|}{ VARIABLES ESENCIALES DEL SISTEMA CLIMÁTICO } \\
\hline \multirow[t]{3}{*}{ ATMÓSFERA } & Superficie & $\begin{array}{l}\text { Temperatura del aire, precipitación, presión atmosférica, balance radiativo, dirección y velo- } \\
\text { cidad del viento, vapor de agua. }\end{array}$ \\
\hline & Altura & $\begin{array}{l}\text { Balance radiativo terrestre (incluyendo radiación solar), temperatura del aire en niveles de altura, } \\
\text { dirección y velocidad del viento, vapor de agua y propiedades de las nubes. }\end{array}$ \\
\hline & Composición & Dióxido de carbono, metano, otros gases de efecto invernadero, propiedades de los aerosoles. \\
\hline \multirow[t]{2}{*}{ OCÉANO } & Superficie & $\begin{array}{l}\text { Temperatura de la superficie del mar, salinidad superficial, nivel del mar, estado del mar, co- } \\
\text { rrientes, color del océano (para la actividad biológica) presión parcial del dióxido de carbono. }\end{array}$ \\
\hline & Sub-superficie & Temperatura, salinidad, corrientes, nutrientes, carbón, fitoplancton, trazadores oceánicos. \\
\hline TRE & \multicolumn{2}{|c|}{$\begin{array}{l}\text { Caudales de ríos, use del agua, agua de subsuelo, niveles de lagos, cubierta de nieve, glaciares y campos de } \\
\text { hielos, permafrost y estacionalidad del suelo congelado, albedo, cubierta terrestre (incluyendo tipo de vegetación), } \\
\text { fracción de absorción de radiación fotosintéticamente activa, índice de área vegetacional, biomasa, humedad } \\
\text { del suelo, alteraciones por fuego. }\end{array}$} \\
\hline
\end{tabular}

del SMOC. De las estaciones de superficie seleccionadas tenemos: Base Esperanza, Base Orcadas, Base Marambio (Argentina); Mawson, Davis, Dome A, Casey (Australia); CMR Eduardo Frei (Chile); Zhongshan (China), Dumont D'Urville (Francia), Neumayer (Alemania); Concordia, Mario Zuchelli (Italia); Swoya (Japón), S.A.N.A.E. AWS (Sudáfrica), Bellingshausen, Novalazarevskaya, Progress, Mirnyj, Vostok (Rusia); Amundsen-Scott, McMurdo (Estados Unidos); Halley, Rothera, Fossil Bluff (Reino Unido); Vernadsky (Ucrania); además de las estaciones automáticas de la Universidad de Wisconsin (EEUU) Larsen Ice Shelf, Butler Island, Sky-Blue, Byrd Station, Mount Siple, Harry, Siple Dome, Gill, Letteau, Relay Station, Dome C II, Whitlock, Marble Point, Marilyn, Ferrell, y Possession Island.
En cuanto a las estaciones de altura (de radiosonda) de la Red Básica, todas forman parte del SMOC, éstas son: Base Marambio (Argentina); Mawson, Davis, Casey (Australia); Dumont D'Urville (Francia); Neumayer (Alemania); SYOWA (Japón); Novalazarevskaja, Mirnyj (Rusia); Amundsen-Scott, McMurdo (EEUU); y Halley (Reino Unido).

\section{EJEMPLOS DE APLICACIÓN}

La Red Básica de observación y la Red del SMOC han permitido, entre otros, realizar estudios de variabilidad y cambio climático. Sin embargo, este no ha sido el único esfuerzo en integrar y acopiar datos climatológicos en la Antártica. READER (REference Antarctic Data for Environmental Research) fue un

Tabla 2. Tendencia de la temperatura anual y estacional ( ${ }^{\circ} \mathrm{C}$ por décadas) en estaciones de la Península Antártica. Números cursivos (en negrillas) son significativos a 10\% (5\%) mientras que aquellos en cursivas y negrillas son significativos al 1\% (Turner y otros 2005). Se agrega estación Frei.

\begin{tabular}{lcccccc}
\hline \multicolumn{1}{c}{ Estación } & Anual & Primavera & Verano & Otoño & Invierno & Periodo \\
\hline Frei & $0.21 \pm 0.35$ & $0.22 \pm 0.46$ & $0.05 \pm 0.09$ & $\mathbf{0 . 3 9} \pm \mathbf{0 . 3 3}$ & $0.62 \pm 2.40$ & $1970-2000$ \\
& $0.11 \pm 0.71$ & $-0.06 \pm 0.87$ & $-0.06 \pm 0.43$ & $0.17 \pm 0.96$ & $0.39 \pm 1.86$ & $1970-2010$ \\
Rothera & $\mathbf{1 . 0 1} \pm \mathbf{1 . 4 2}$ & $1.06 \pm 1.53$ & $0.36 \pm 0.57$ & $\mathbf{1 . 3 7} \pm \mathbf{1 . 4 6}$ & $1.73 \pm 2.79$ & $1978-2000$ \\
& $\mathbf{0 . 6 9} \pm \mathbf{1 . 3 1}$ & $\mathbf{0 . 7 6} \pm \mathbf{1 . 5 1}$ & $0.18 \pm 0.56$ & $\mathbf{0 . 6 7} \pm \mathbf{1 . 3 2}$ & $1.09 \pm 2.87$ & $1978-2010$ \\
Faraday & $\mathbf{0 . 5 6} \pm \mathbf{0 . 4 3}$ & $0.25 \pm 0.44$ & $\mathbf{0 . 2 4} \pm \mathbf{0 . 1 7}$ & $\mathbf{0 . 6 3} \pm \mathbf{0 . 6 0}$ & $\mathbf{1 . 0 9} \pm \mathbf{0 . 8 8}$ & $1951-2000$ \\
& $\mathbf{0 . 5 5} \pm \mathbf{1 . 2 6}$ & $\mathbf{0 . 3 9} \pm \mathbf{1 . 4 3}$ & $\mathbf{0 . 2 3} \pm \mathbf{0 . 6 3}$ & $\mathbf{0 . 5 2} \pm \mathbf{1 . 6 2}$ & $\mathbf{1 . 0 6} \pm \mathbf{2 . 7 9}$ & $1951-2010$ \\
Bellingshausen & $\mathbf{0 . 3 5} \pm \mathbf{0 . 4 6}$ & $-0.10 \pm 0.47$ & $\mathbf{0 . 3 0} \pm \mathbf{0 . 2 0}$ & $\mathbf{0 . 5 1} \pm \mathbf{1 . 0 5}$ & $0.58 \pm 0.97$ & $1969-2000$ \\
& $0.26 \pm 0.75$ & $0.10 \pm 0.91$ & $0.13 \pm 0.45$ & $0.33 \pm 1.04$ & $0.48 \pm 1.89$ & $1969-2010$ \\
Esperanza & $0.41 \pm 0.42$ & $-0.07 \pm 0.57$ & $\mathbf{- 0 . 0 7} \pm \mathbf{0 . 5 7}$ & $\mathbf{0 . 4 3} \pm \mathbf{0 . 3 4}$ & $0.51 \pm 0.82$ & $1961-2000$ \\
& $\mathbf{0 . 3 3} \pm \mathbf{1 . 0 8}$ & $0.14 \pm 1.52$ & $\mathbf{0 . 1 4} \pm \mathbf{1 . 5 2}$ & $0.37 \pm 0.80$ & $0.32 \pm 2.20$ & $1961-2010$ \\
Marambio & $<90 \%$ & $-0.80 \pm 10.5$ & $<90 \%$ & $<90 \%$ & $0.81 \pm 1.53$ & $1971-2000$ \\
Orcadas & $\mathbf{0 . 4 5} \pm \mathbf{1 . 1 7}$ & $0.34 \pm 1.87$ & $\mathbf{0 . 5 1} \pm \mathbf{0 . 8 7}$ & $0.62 \pm 2.43$ & $0.40 \pm 2.72$ & $1971-2010$ \\
& $\mathbf{0 . 2 0} \pm \mathbf{0 . 1 0}$ & $\mathbf{0 . 1 5} \pm \mathbf{0 . 1 4}$ & $\mathbf{0 . 1 5} \pm \mathbf{0 . 0 6}$ & $\mathbf{0 . 2 1} \pm \mathbf{0 . 1 6}$ & $\mathbf{0 . 2 7} \pm \mathbf{0 . 2 4}$ & $1904-2000$ \\
\hline
\end{tabular}


proyecto del Comité de Científico de Investigación Antártica (SCAR: Scientific Committee on Antarctic Research) con el propósito de crear una base de datos de alta calidad de las estaciones antárticas. Las mismas que forman parte del SMOC. Datos históricos y actualizados mes a mes se pueden encontrar en http://www.antarctica.ac.uk/met/READER/. A partir de ellos se han realizado estudios sobre el comportamiento de la temperatura del aire superficial y en la tropósfera media en la Antártica, como son los trabajos de Turner et al. (2005) y Turner et al. (2006), respectivamente.

La Tabla 2 resume las tendencias lineales de las temperaturas media anual y estacional de las estaciones meteorológicas ubicadas en la Península Antártica. Estos datos fueron extraídos del estudio de Turner et al. (2005) y se incluye los datos de la estación Eduardo Frei. En términos anuales hay un calentamiento de entre $0.20 \mathrm{a} 1.01^{\circ} \mathrm{C}$ por década, donde el análisis estacional revela que son los meses de otoño $e$ invierno los que muestran el mayor calentamiento. Algunas estaciones muestran un leve enfriamiento en primavera lo que indica la alta variabilidad espacial de la temperatura aún en estaciones cercanas como Rothera y Faraday respecto de Frei/Bellingshausen. Los resultados de Turner y otros (2005) indican además, que al menos en superficie, el calentamiento está restringido al sector norte de la península y es más significativo en el lado oeste, siendo la estación Rothera la que mayor calentamiento registra, mientras que el resto del continente no presenta calentamiento. Esto confirma los estudios anteriores realizados por King (1994), King \& Harangozo (1998), Quintana \& Carrasco (2000), Carrasco \& González (2007), Marshal et al. (2002) y King \& Comiso (2003), entre otros; que mostraron el fuerte calentamiento en la Península Antártica que ha estado ocurriendo desde mediado del siglo XX y que no tiene parangón en otra parte del planeta. Además, la Tabla 2 la actualiza las tendencias hasta el año 2010. Note que en la mayoría de los casos de las tendencias ampliadas hasta el 2010 muestran valores menores a los períodos que llegan hasta el año 2000 que abarca el estudio de Turner $y$ otros (2005). Esto refleja un menor calentamiento en la última década comparado a aquel observado en la segunda mitad del siglo XX. Incluso algunas estaciones (Frei, Marambio, Esperanza) revelan una pendiente anual negativa en el periodo 2001-2010 aunque no estadísticamente significativo.
Por otra parte, el estudio de Turner y otros (2006) indica que en la troposfera media sí se observa un calentamiento generalizado en la Antártica durante el invierno. Los resultados muestran un aumento estadísticamente significativo de la temperatura de la troposfera media de 0.5 a $0.7^{\circ}$ C por década para el período 1971-2003. En este orden, la Figura 2 muestra el comportamiento de la temperatura del aire a $850 \mathrm{hPa}(\sim 1.000-1.200 \mathrm{~m})$, que representa la atmósfera cercana de la superficie pero alejada de su influencia directa; a $500 \mathrm{hPa}(\sim$ $4.900-5.200 \mathrm{~m}$ ) que representa la altura media de la troposfera y a $100 \mathrm{hPa}(\sim 15.500-16.000$ m) que corresponde a la atmósfera por sobre la tropopausa (la estratosfera baja). Los datos fueron recolectados por los radiosondeos realizados en las estaciones de Faraday y Bellingshausen (www. antarctica.ac.uk), los cuales operaron regularmente entre los años 1955-1982 y 1970-1997, respectivamente. El traslape de años (1970-1982) permitió realizar correlaciones mensuales entre ambas serie de datos y obtener un factor de corrección para extender la serie de Bellingshausen hasta 1956 en base al dato de Faraday. De este modo se obtuvo el comportamiento de la atmósfera baja, media y superior en el sector norte de la Península Antártica para el periodo 1955-1996. A igual que en superficie los resultados muestran un calentamiento estadísticamente significativo ( $1 \%$ de confianza) de $0.35^{\circ} \mathrm{C}$ por década en los 850 y $500 \mathrm{hPa}$ y un leve enfriamiento en la estratosfera de $0.17^{\circ} \mathrm{C}$ por década, aunque no estadísticamente significativo.

\section{CONCLUSIÓN}

El SMOC es una iniciativa que ha logrado mantener una red básica de observación del clima y comprometer a servicios e instituciones, desde el nivel nacional al internacional, en un esfuerzo colaborativo para tener acceso a bases de datos que permitan realizar estudios de cambio climáticos y otros. En particular, en la Antártica las iniciativas del SCAR y los esfuerzos del British Antarctic Survey, de la Universidad de Wisconsin y de otros tantos organismos nacionales, han permitido la creación de bases de datos para el estudio medio ambiental. Por solo nombrar algunas direcciones de páginas web, se señalan las siguientes en donde el lector puede explorar por datos e información: 

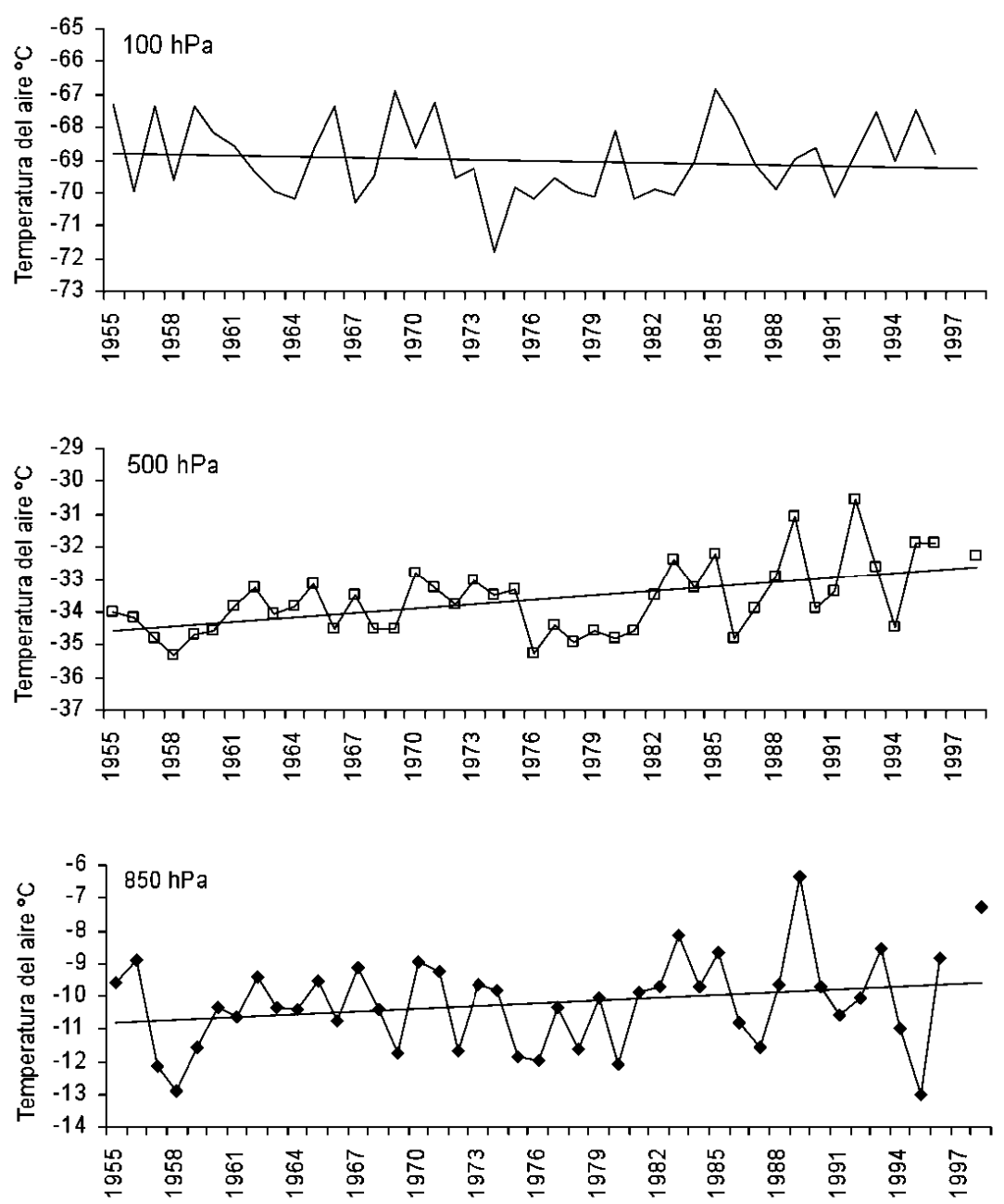

Fig. 2. Temperatura media anual a $850 \mathrm{hPa}, 500 \mathrm{hPa}$ y $100 \mathrm{hPa}$ obtenido de los radiosondas de las estaciones Bellingshausen y Faraday.

http://bprc.osu.edu/polar_pointers/

http://www.antarctica.ac.uk/bas_research/ data/index.php

http://amrc.ssec.wisc.edu/

http://www.esrl.noaa.gov/psd/data/reanalysis/ reanalysis.shtml

http://lance-modis.eosdis.nasa.gov/imagery/ subsets/?project=antarctica

Los resultados acerca del comportamiento de la temperatura del aire, son ejemplos de la colaboración internacional para la investigación del cambio climático en base a la Red de estaciones de observación atmosférica en la Antártica. La nece- sidad de continuar y asegurar el funcionamiento de las estaciones antárticas para el monitoreo de las variables esenciales del sistema climático es evidente para los estudios, no sólo de la variabilidad y el cambio climático, sino también para la predicción diaria y estacional. La Antártica no es un continente aislado (Carrasco 2008). Su ubicación geográfica no sólo influye el comportamiento meteorológico regional en las zonas polares sino que también ejerce una influencia en las características del clima del Hemisferio Sur. Juega un papel importante en la formación y/o localización de la difluencia de la corriente en chorro (zonas de vientos fuertes el altura) en las cercanías de Nueva Zelanda, influenciando 
así la meteorología y climatología del Hemisferio Sur. La variabilidad interanual que se observa en la Antártica está vinculada a mecanismos de teleconexión como El Niño Oscilación del Sur (Turner 2004) y la Oscilación Antártica (AO). En conclusión, la Antártica regional y globalmente es un continente integrado al resto del planeta que influye en la circulación de la atmósfera (y el océano) y responde al cambio climático, así como también es afectado por los cambios atmosféricos y variabilidad climática (y oceánicos) de mecanismos naturales como El Niño y está expuesto a los efectos de los cambios climáticos de origen antropogénico (Carrasco 2008), y es esencial la continuación y la implementación de programas de observación sistemática de variables climáticas.

\section{AGRADECIMIENTOS}

El autor agradece a la Dirección Meteorológica de Chile dependiente de la Dirección General de Aeronáutica Civil por el constante apoyo al Sistema Mundial de Observación del Clima y a mi persona como punto focal en Chile. Al Proyecto Anillo Conicyt-Bicentenario ARTG02-2006 "Stability and recent behaviour of glaciers in Antarctic Peninsula the interactions with ice shelves", que permitió análisis de datos mostrados que parcialmente se incluyen en Tabla 2 y Figura 2.

\section{LITERATURA CITADA}

Carrasco, J.F. 2008. La Antártica no es un continente aislado. Boletín Antártico Chileno. 27(2):12-14

Carrasco, J.F. \& M. González 2007. Climatología de la Península Antártica y de la Base Eduardo Frei Montalva. Editado y Publicado por la Dirección Meteorológica de Chile, pp. 105 (Disponible en www.meteochile.gob.cl/ climatologia).

GCOS, 2007: Global Climate Observing System Ensuring the Availability of Global Observations for Climate. 18 pp.

King, J.C. 1994: Recent climate variability in the vecinity of the Antarctic Peninsula. International Journal of Climatology, 14:357-369
King, J.C. \& J.C. Comiso 2003. The spatial coherence of interannual temperature variations in the Antarctic Peninsula. Geophysical Research Letter, 30(2): 1040, doi:10.1029/2002GL015580.

King, J.C. \& S.A. Harangozo 1998. Climate change in the western Antarctic Peninsula 1945-present: observations and possible causes. Annals of Glaciology, 27:571-575

Marshal, G.J., V. Lagun, T.A. \& Lachlan-Cope 2002. Change in Antarcitic Peninsula tropospheric temperatures from 1956-99; a synthesis of observations and reanalysis data. Internatilnal Journal of Climatology, 22:291-310

Meehl, G.A., T.F. Stocker, W.D. Collins, P. Friedlingstein, A.T. Gaye, J.M. Gregory, A. Kitoh, R. Knutti, J.M. Murphy, A. Noda, S.C.B. Raper, I.G. Watterson, A.J. Weaver \& Z.C. Zhao 2007. Global Climate Projections. In: Climate Change 2007: The Physical Science Basis. Contribution of Working Group I to the Fourth Assessment Report of the Intergovernmental Panel on Climate Change [Solomon, S., D. Qin, M. Manning, Z. Chen, M. Marquis, K.B. Averyt, M. Tignor and H.L. Miller (eds.)]. Cambridge University Press, Cambridge, United Kingdom and New York, NY, USA.

Quintana, J. \& J.F. Carrasco 2000. Temperature and Precipitation behavior during 1961-1998 period at the northern tip of the Antarctic Peninsula En Sixth International Conference on Southern Hemisphere Meteorology and Oceanography, 234-235 pp. Amer. Meteo. Soc.

Turner, J. 2004. Review: The El Niño-Southern Oscillation and Antarctica. International Journal of Climatology, 24:1-31

Turner, J., S.R. Colwell, G.J. Marshall, T.A. LachlanCope, A.M. Carleton, P.D. Jones, V. Lagun, P.A. Reid \& S. Iagovkina 2005. Antarctic climate change during the last 50 years. International Journal of Climatology, 25:279-294

Turner, J., T.A. Lachlan-Cope, S. Colwell, G.J. Marshall, \& W.M. Connolley 2006: Significant warming of the Antarctic winter troposphere. Science, 311:1914-1917 
J. CARRASCO 